\title{
Relativity and Radiation Balance for the Classical Hydrogen Atom in Classical Electromagnetic Zero-Point Radiation
}

\author{
Timothy H. Boyer \\ Department of Physics, City College of the City \\ University of New York, New York, New York 10031
}

\begin{abstract}
When teaching modern physics, every instructor must deal with the apparent failure of classical physics to prevent the radiation collapse of the nuclear atom. Here we review the understanding of the classical hydrogen atom in classical electromagnetic zero-point radiation, and emphasize the importance of special relativity. The crucial missing ingredient in earlier calculational attempts (both numerical and analytic) is the use of valid approximations to the full relativistic analysis. It is pointed out that the nonrelativistic time Fourier expansion coefficients given by Landau and Lifshitz are in error as the electromagnetic description of a charged particle in a Coulomb potential, and, because of this error, Marshall and Claverie's conclusion regarding the failure of radiation balance is invalid. Rather, using Marshall and Claverie's calculations, but restricted to lowest nonvanishing order in the orbital eccentricity (where the nonrelativistic orbit is a valid approximation to the fully relativistic electromagnetic orbit) radiation balance for classical electromagnetic zero-point radiation is shown to hold at the fundamental frequencies and associated first overtones.
\end{abstract}




\section{INTRODUCTION}

\section{A. "Modern Physics" and the Apparent Failure of "Classical Physics"}

In introductory classes in modern physics, an instructor often reviews the alleged failures of classical physics to account for the blackbody radiation spectrum and the stability of the hydrogen atom, among other phenomena. The subject is often taught as though the conclusions were unambiguous and closed; quantum theory is the only alternative. However, the matter may not be so completely closed as we had been led to think. Indeed, some of the claims made in the modern physics texts[1] regarding the failures of classical physics are actually wrong. In a previous historical review, the blackbody radiation problem was treated, [2] and it was pointed out that the historical arguments are modified in the presence of zero-point radiation in such a way as to give natural classical explanations for the Planck spectrum. In this article, we return to the problem of the collapse of the nuclear atom in classical electromagnetism which appeared to be settled in the first quarter of the 20th century. As suggested here, the problem seems complicated, and instructors actually need to be cautious when describing the classical physics.

\section{B. History of the Classical Hydrogen Atom Problem}

Contrary to the statements in the modern physics texts, [1] it has been suggested for over half a century that it may be possible to understand the apparently radiationless ground state of the classical hydrogen atom as arising from a Brownian motion of the electron in classical electromagnetic zero-point radiation. [3] [4] The classical theory of which this idea forms a crucial part is often referred to as "stochastic electrodynamics." During the 1960s, this hopeful understanding was encouraged by easy calculations involving either perfect conductors or harmonic oscillators in classical zero-point radiation, which led to classical derivations in agreement with nature for Casimir forces, van der Waals forces, specific heats of solids, diamagnetic behavior, and the Planck blackbody spectrum. [5] [6] However, in the 1970s, this hope was discouraged by calculations which indicated that nonlinear systems did not preserve radiation balance for zero-point radiation, but rather pushed the zero-point spectrum toward the Rayleigh-Jeans spectrum. [7] [8] Finally in 1980, the hope of a classical understanding of hydrogen in zero-point radiation seemed futile because of sophisticated 
analytic work by Marshall and Claverie.[9] These authors apparently showed that a nonrelativistic charged particle in a Coulomb potential in zero-point radiation did not preserve radiation balance for zero-point radiation. Further analysis indicated that the electron picked up too much energy from the zero-point radiation, and so the classical hydrogen atom was self-ionizing. [10] Thus the atomic collapse problem of the early twentieth century was replaced by a new problem where zero-point radiation provided too much energy. Marshall and Claverie regarded their calculations as being consistent with the earlier nonlinear calculations of the 1970s leading to the Rayleigh-Jeans spectrum for radiation balance, but depreciated the previous suggestions that the use of relativistic scatterers might be the missing ingredient leading back to the hoped-for stability and radiation balance for hydrogen in zero-point radiation.

The gloom surrounding stochastic electrodynamics was suddenly lifted in 2003 by nonrelativistic numerical simulations carried out by Cole and Zou for the classical hydrogen atom in classical zero-point radiation.[11] Using no adjustable parameters, they showed that the probability distribution for the position of the nonrelativistic electron seemed to be approaching that given by the Schroedinger equation ground state. However, in 2015, a damper on the enthusiasm arrived with new and longer calculations by Nieuwenhuizen and Liska which indicated disagreement with the Schroedinger ground state and which emphasized the self-ionizing aspects of the simulation calculations. [12] Although it had been noted that relativity would modify the plunging orbits where the self-ionization occurred, [13] Nieuwenhuizen and Liska dismissed the role of relativity; they carried out further calculations suggesting that relativity made only insignificant changes in their simulations. [14]

\section{Evidence for the Importance of Relativity}

Recently the outlook has changed yet again due to the first-ever scattering calculation involving a system which can be regarded as relativistic. 15] The first important observation for the new scattering calculation is that we can think of a nonrelativistic calculation as a valid approximation to the accurate relativistic calculation in the parameter range where both calculations agree. Specifically, a particle in a harmonic potential taken in the smallamplitude limit, can be regarded as approximately relativistic, since when the velocity is small, $v<<c$, the particle momentum change $d \mathbf{p} / d t$ and the particle kinetic energy cal- 
culated nonrelativistically agree with the accurate relativistic calculations, with the first corrections starting at order $(v / c)^{2}$. The second needed aspect for a relativistic scattering calculation was provided by Huang and Batelaan's emphasis that when the oscillation amplitude of a harmonic oscillator is non-zero, then the oscillator will interact with radiation not only at the fundamental frequency but at all its harmonics. [16] A purely harmonic oscillator of finite amplitude of oscillation will produce not only dipole radiation but also quadrupole radiation and indeed radiation at all the harmonics. Thus the purely harmonic oscillator of finite amplitude but with $v<<c$ provides a valid approximation to a relativistic system but also connects the system to radiation at several frequencies. Here finally was a simple mechanical system where radiation equilibrium is not forced by the mechanical system but rather is determined by the multipole electromagnetic interactions connected to the full space and time dependence of the radiation field. And indeed, calculations showed that for this relativistic system, radiation equilibrium required a relativistic spectrum of random electromagnetic radiation, and not the Rayleigh-Jeans spectrum. [15] Electromagnetism is a relativistic theory and Lorentz-invariant classical zero-point radiation should fit with electromagnetic theory. For this relativistic oscillator system, radiation balance held for a Lorentz-invariant spectrum of random classical radiation, indeed for classical electromagnetic zero-point radiation. In this case, relativity was indeed the missing ingredient for radiation balance.

\section{Reconsideration of the Classical Hydrogen Ground State}

This result involving a relativistic system and classical zero-point radiation has changed the outlook for classical mechanical systems in random radiation. In the present article, we will review briefly the ideas of classical zero-point radiation, and then we will look again at a charged particle in a Coulomb potential in classical zero-point radiation. Naively, we tend to think that nonrelativistic approximations should always be valid for a charged particle in a Coulomb potential. However, we will point out that some of the earlier work on hydrogen does not provide nonrelativistic limits which are consistent with relativistic behavior. We will reconsider the analytic calculations of Marshall and Claverie which were carried out in 1980. Here we will take as valid precisely those aspects which are consistent with the relativistic limit, and only those aspects. In contradiction with the earlier claims 
that radiation balance did not hold, we will show that Marshall and Claverie's calculations give radiation balance at both the fundamental orbital frequencies and the associated first overtones for calculations involving terms through first order in the orbital eccentricity where the nonrelativistic calculations provide a valid approximation to the actual relativistic electromagnetic situation. Also, we point out that nonrelativistic numerical simulations may underestimate the importance of relativistic corrections, especially in regions of large eccentricity.

\section{BASIC IDEAS OF CLASSICAL ELECTRODYNAMICS WITH CLASSICAL ELECTROMAGNETIC ZERO-POINT RADIATION}

\section{A. Elements Missing from Classical Theory in 1900}

At the turn of the 20th century, physicists did not appreciate two crucial aspects of classical theory. [2] 1) Classical electrodynamics is a relativistic theory and any valid analysis must be consistent with relativity. 2) Lorentz-invariant classical electromagnetic zero-point radiation is present in nature.

Indeed, there is a profound conflict within classical physics which has not been appreciated. Classical electrodynamics always connects charges to radiation so that an accelerating charge emits electromagnetic radiation. On the other hand, this connection of a particle with its field is absent in classical mechanics where a (frictionless) particle in a potential may oscillate indefinitely without losing energy. Because physicists were so used to the ideas of classical mechanics in the early 20th century, they attempted to construct a theory which broke the connection between a charged particle and its radiation field. Thus in his description of old quantum theory, Born writes, "The endeavour to retain the classical mechanics as far as possible having proved to be a fertile method, we take as our first requirement that the stationary states of an atomic system shall be calculated as far as possible, in accordance with the laws of classical mechanics, but the classical theory of radiation is disregarded." [17] 


\section{B. Classical Zero-Point Radiation}

The disregard for the classical theory of radiation has been countered and transformed by the idea of classical electromagnetic zero-point radiation. The first careful and extensive effort to treat problems of atomic physics entirely within classical electromagnetism was made by Marshall[3] beginning in 1963. Marshall looked for the classical electromagnetic radiation field which would give an oscillator the same average energy as appeared for a quantum oscillator in its ground state. This corresponded to assuming that nature contained random classical radiation at the zero of temperature with an average energy per normal mode given by $U_{\text {radzp }}(\omega)=(1 / 2) \hbar \omega$.

Random classical radiation can be written in the form used at the end of the 19th century as a sum over plane waves with random phases in a large cubic box with sides of length $a$,

$$
\begin{gathered}
\mathbf{E}(\mathbf{r}, t)=\sum_{\mathbf{k}, \lambda} \widehat{\epsilon}(\mathbf{k}, \lambda)\left(\frac{8 \pi U_{\operatorname{rad}}(\omega)}{a^{3}}\right)^{1 / 2} \frac{1}{2}\{\exp [i \mathbf{k} \cdot \mathbf{r}-i \omega t+i \theta(\mathbf{k}, \lambda)]+c c\} \\
\mathbf{B}(\mathbf{r}, t)=\sum_{\mathbf{k}, \lambda} \widehat{\mathbf{k}} \times \widehat{\epsilon}(\mathbf{k}, \lambda)\left(\frac{8 \pi U_{\operatorname{rad}}(\omega)}{a^{3}}\right)^{1 / 2} \frac{1}{2}\{\exp [i \mathbf{k} \cdot \mathbf{r}-i \omega t+i \theta(\mathbf{k}, \lambda)]+c c\}
\end{gathered}
$$

where the sum over the wave vectors $\mathbf{k}=\widehat{x} 2 \pi l / a+\widehat{y} 2 \pi m / a+\widehat{z} 2 \pi n / a$ involves integers $l, m, n=0, \pm 1, \pm 2, \ldots$ running over all positive and negative values, there are two polarizations $\widehat{\epsilon}(\mathbf{k}, \lambda), \lambda=1,2$, and the random phases $\theta(\mathbf{k}, \lambda)$ are distributed uniformly over the interval $(0,2 \pi]$, independently for each wave vector $\mathbf{k}$ and polarization $\lambda$. The notation "cc" refers to the complex conjugate. The energy per normal mode at radiation frequency $\omega=c k$ is given by $U_{\text {rad }}(\omega)$, and we have assumed that the radiation spectrum is isotropic.

\section{Time Correlation Function for the Electric Fields}

The correlation functions of the electric and magnetic fields can be obtained by averaging over the random phases $\theta(\mathbf{k}, \lambda)$. Thus for the time-correlation function for the electric field 
at a single point in space $\mathbf{r}=0$, we find

$$
\begin{aligned}
& \left\langle E_{i}(0, t) E_{j}(0, t+\tau)\right\rangle \\
& =\sum_{\mathbf{k}, \lambda} \sum_{\mathbf{k}^{\prime}, \lambda^{\prime}} \epsilon_{i}(\mathbf{k}, \lambda) \epsilon_{j}\left(\mathbf{k}^{\prime}, \lambda^{\prime}\right)\left(\frac{8 \pi U_{r a d}(\omega)}{a^{3}}\right)^{1 / 2}\left(\frac{8 \pi U_{r a d}\left(\omega^{\prime}\right)}{a^{3}}\right)^{1 / 2} \frac{1}{4} \\
& \times\left\langle\{\exp [-i \omega t+i \theta(\mathbf{k}, \lambda)]+c c\}\left\{\exp \left[-i \omega^{\prime}(t+\tau)+i \theta\left(\mathbf{k}^{\prime}, \lambda^{\prime}\right)\right]+c c\right\}\right\rangle \\
& =\sum_{\mathbf{k}, \lambda} \epsilon_{i}(\mathbf{k}, \lambda) \epsilon_{j}(\mathbf{k}, \lambda)\left(\frac{8 \pi U_{r a d}(\omega)}{a^{3}}\right) \frac{1}{4}\{\exp [i \omega \tau]+c c\}
\end{aligned}
$$

since averaging over the random phases involves

$$
\left\langle\exp [i \theta(\mathbf{k}, \lambda)] \exp \left[-i \theta\left(\mathbf{k}^{\prime}, \lambda^{\prime}\right)\right]\right\rangle=\delta_{\mathbf{k k}^{\prime}} \delta_{\lambda \lambda^{\prime}}
$$

If the box for the periodic boundary conditions is taken as very large, the then sums can be

replaced by integrals, $\sum_{\mathbf{k}} \rightarrow \int[a /(2 \pi)]^{3} d^{3} k$. Also, since the radiation is assumed isotropic, the sum over polarizations $\sum_{\lambda} \epsilon_{i}(\mathbf{k}, \lambda) \epsilon_{j}(\mathbf{k}, \lambda)=\delta_{i j}-k_{i} k_{j} / k^{2}$ can be replaced by $\delta_{i j} 2 / 3$. Thus the electric field correlation function (3), with $B_{E}(\tau)$ defined as $\left\langle E_{i}(0, t) E_{j}(0, t+\tau)\right\rangle=$ $\delta_{i j} B_{E}(\tau)$, becomes

$$
\begin{aligned}
\left\langle E_{i}(0, t) E_{j}(0, t+\tau)\right\rangle & =\delta_{i j} \int \frac{d^{3} k}{\pi^{2}} \frac{2}{3} U_{\text {rad }}(\omega) \frac{1}{4}\{\exp [i \omega \tau]+c c\} \\
& =\delta_{i j} \int_{-\infty}^{\infty} d \omega \frac{2}{3 \pi c^{3}} \omega^{2} U_{\text {rad }}(\omega) \exp [i \omega \tau]
\end{aligned}
$$

where we have integrated over angles to give a factor of $4 \pi$ and have extended the $\omega$ integral to $-\infty$ by using the complex conjugate term. We define the spectrum $S_{E}(\omega)$ as

$$
\begin{aligned}
S_{E}(\omega) & =\int_{-\infty}^{\infty} d \tau B_{E}(\tau) \exp [-i \omega \tau] \\
& =\int_{-\infty}^{\infty} d \tau \exp [-i \omega \tau] \int_{-\infty}^{\infty} d \omega^{\prime} \frac{2}{3 \pi c^{3}} \omega^{\prime 2} U_{r a d}\left(\omega^{\prime}\right) \exp \left[i \omega^{\prime} \tau\right] \\
& =\frac{2}{3 c^{3}} 2 \omega^{2} U_{r a d}(\omega)
\end{aligned}
$$

If we consider zero-point radiation where $U_{\text {radzp }}(\omega)=(1 / 2) \hbar \omega$, then the spectrum is

$$
S_{E \mathrm{zp}}(\omega)=\frac{2}{3 c^{3}} \hbar \omega^{3} .
$$

\section{Dipole Oscillator in Random Classical Radiation}

The behavior of a harmonic oscillator system (of natural frequency $\omega_{0}$, mass $m$, and charge $e$ ) in random radiation, treated in the dipole approximation, follows from Newton's 
second law as[3] [5]

$$
m \ddot{x}=-m \omega_{0}^{2} x-m \tau \dddot{x}+e E_{x}(0, t)
$$

where the term in $\dddot{x}$ involves the radiation reaction force with $\tau=\left(2 e^{2}\right) /\left(3 m c^{3}\right)$. The steady-state solution takes the form

$$
x(t)=\frac{e}{m} \sum_{\mathbf{k}, \lambda} \epsilon_{x}(\mathbf{k}, \lambda)\left(\frac{8 \pi U_{r a d}(\omega)}{a^{3}}\right)^{1 / 2} \frac{1}{2}\left\{\frac{\exp \{i[\mathbf{k} \cdot \mathbf{r}-\omega t+\theta(\mathbf{k}, \lambda)]\}}{-\omega^{2}+\omega_{0}^{2}+i \tau \omega^{3}}+c c\right\} .
$$

The successful calculations, involving Casimir forces, van der Waals forces, specific heats of solids, diamagnetism, and the blackbody radiation, [5] [6] all involved either purely electromagnetic fields or else harmonic oscillator systems.

\section{E. Radiation Balance}

When treated in the electric dipole approximation, charged mechanical systems have a standard interaction with radiation. Energy absorption occurs through the forcing term $e \mathbf{E}(0, t) \cdot \mathbf{v}$ while energy emission occurs according to Larmor's formula for electric dipole oscillation. Linear oscillator systems, treated in the electric dipole approximation, come to equilibrium when the average energy of the mechanical oscillator equals the average energy of the normal modes of the radiation field at the natural frequency $\omega_{0}$ of the linear oscillator. Furthermore, in the dipole approximation, linear oscillator systems will scatter radiation, but do not change the frequency spectrum of the radiation. Thus since classical electromagnetic zero-point radiation is assumed isotropic, the harmonic oscillators following Eqs. (8) and (9) leave the radiation pattern of classical zero-point radiation undisturbed.[18] Linear oscillators treated in the dipole approximation preserve radiation balance in any isotropic spectrum of random radiation, and, in equilibrium, an experimenter would not find any net radiation emission or absorption at any frequency.

However, as pointed out in Jackson's text, [19] "Appreciable radiation in multiples of the fundamental (frequency) can occur because of relativistic effects ... or it can occur if the components of velocity are not sinusoidal, even though periodic." Nonlinear oscillators have time Fourier components of velocity (and hence of radiation) at all multiples of the fundamental oscillation frequency. Thus nonlinear oscillators, treated in the electric dipole

approximation, can absorb energy at one frequency and reradiate the energy at a different frequency, and so can indeed change the frequency spectrum of the ambient radiation. It was 
found that a nonrelativistic nonlinear oscillator did not preserve radiation balance for classical zero-point radiation, but rather tended to push the spectrum toward the Rayleigh-Jeans spectrum where the same energy per normal mode exists at all frequencies. [7] [8] Thus, in classical zero-point radiation, the nonlinear oscillators absorbed radiation at high frequencies where the energy per normal mode was larger, and reradiated this energy at low frequencies where the energy per normal mode was smaller, so as to tend to equalize the energy per normal mode at all frequencies. Indeed, within the narrow-line-width approximation, such scattering calculations for nonlinear systems had been carried out (but only partly published) by van Vleck in 1924.[20]

\section{REFINEMENTS IN THE MATHEMATICAL ANALYSIS}

\section{A. Use of Action-Angle Variables in Mechanical Systems}

The interest in nonlinear scattering systems brought attention back to the use of actionangle variables in classical mechanics associated with old quantum theory. [8] [17] The action variables are adiabatic invariants, and so are ideal for use in perturbation calculations where the mechanical system is weakly perturbed by electromagnetic radiation. The calculations by van Vleck[20] in 1924 and by the physicists interested in zero-point radiation in the 1970s involved a purely dipole connection between the mechanical system and radiation. Thus the purely mechanical system of a particle of mass $m$ in a potential $V(\mathbf{r})$ was assumed described by the mechanical equation

$$
\frac{d \mathbf{p}}{d t}=-\nabla V(\mathbf{r})
$$

Then action-angle variables $w_{i}, J_{i}, i=1,2,3$ were introduced so that the particle position could be described as a multiply periodic system with expansion coefficients $\mathbf{D}_{l m n}\left(J_{1}, J_{2}, J_{3}\right)$ such that

$$
\mathbf{r}=\sum_{l m n} \mathbf{D}_{l m n}\left(J_{1}, J_{2}, J_{3}\right) \exp \left[i\left(l w_{1}+m w_{2}+n w_{3}\right)\right]
$$

where the integers $l, m, n=0, \pm 1, \pm 2, \ldots$, and where the angle variables advance uniformly in time

$$
w_{i}=\omega_{i} t+\beta_{i}
$$

The (angular) frequencies $\omega_{i}$ are functions of the action variables, $\omega_{i}=\omega_{i}\left(J_{1}, J_{2}, J_{3}\right)$, and the action variables $J_{i}, i=1,2,3$ are constant in time. 


\section{B. Mechanical System Perturbed by Random Radiation}

If the particle in the potential $V(\mathbf{r})$ carries a charge $e$ in the presence of random radiation, then the equation of motion in the dipole approximation becomes a generalized Langevin equation

$$
\frac{d \mathbf{p}}{d t}=-\nabla V(\mathbf{r})+\frac{2 e^{2}}{3 c^{3}} \dddot{\mathbf{r}}+e \mathbf{E}(0, t),
$$

where the term $\left[2 e^{2} /\left(3 c^{3}\right)\right] \dddot{\mathbf{r}}$ represents the force due to dipole radiation damping and the term $e \mathbf{E}(0, t)$ represents the force due to the random electric field, again taken in the dipole approximation $e \mathbf{E}(\mathbf{r}, t) \approx e \mathbf{E}(0, t)$.

\section{Quasi-Markov Process and the Fokker-Planck Equation}

The charged particle whose motion is described by Eq. (13) will both lose energy by radiation emission and absorb energy from the ambient radiation. If the magnitude $e$ of the charge is small, then the particle will carry out many mechanical oscillations before there is significant energy exchange with the radiation field. In this "narrow-line-width" approximation, Marshall[21] and Marshall and Claverie[22] showed that the charged particle can be regarded as carrying out a quasi-Markov process described by a Fokker-Planck equation for the probability distribution $P\left(J_{1}, J_{2}, J_{3}, t\right)$ of the action variables,

$$
\frac{\partial}{\partial t} P\left(J_{1}, J_{2}, J_{3}, t\right)=\sum_{i} \frac{\partial}{\partial J_{i}}\left(-A_{i} P\left(J_{1}, J_{2}, J_{3}, t\right)+\sum_{j} B_{i j} \frac{\partial}{\partial J_{j}} P\left(J_{1}, J_{2}, J_{3}, t\right)\right) .
$$

Here the terms $A_{i}$ are referred to as "drift coefficients" and the terms $B_{i j}$ as "diffusion coefficients." In work in 1924, Van Vleck[20] carried out separate calculations for the mean change in the mechanical action variables $J_{i}$ due to emission of radiation by the charge in a small time $\delta t$, the mean change in the action variables due to absorption of radiation by the charge during $\delta t$, and the mean squares of the changes in the action variables during the time $\delta t$. Marshall and Claverie [9] [21] [22] gave the average loss in the drift coefficients $A_{i}$, and combined the average gain terms together with the mean-square gain into the diffusion coefficients $B_{i j}$. Furthermore they showed that the drift coefficients and diffusion coefficients could be calculated from the unperturbed motion of the particle as

$$
A_{i}=\sum_{j}\left\langle\frac{2 e^{2}}{3 c^{3}} \dddot{x}_{j} \frac{\partial J_{i}}{\partial p_{j}}\right\rangle=\sum_{j}\left\langle\frac{2 e^{2}}{3 c^{3}} \dddot{x}_{j} \frac{\partial x_{j}}{\partial w_{i}}\right\rangle
$$


and

$$
\begin{aligned}
B_{i j} & =\frac{e^{2}}{2} \int_{-\infty}^{\infty} B_{E}(\tau) \sum_{k}\left\langle\frac{\partial J_{i}}{\partial p_{k}}(t) \frac{\partial J_{i}}{\partial p_{k}}(t+\tau)\right\rangle d \tau \\
& =\frac{e^{2}}{2} \int_{-\infty}^{\infty} B_{E}(\tau) \sum_{k}\left\langle\frac{\partial x_{k}}{\partial w_{i}}(t) \frac{\partial x_{k}}{\partial w_{j}}(t+\tau)\right\rangle d \tau,
\end{aligned}
$$

where

$$
\delta_{i j} B_{E}(\tau)=\left\langle E_{i}(0, t) E_{j}(0, t+\tau)\right\rangle
$$

the brackets refer to averaging over time $t$, and use has been made of the relationship

$$
\frac{\partial J_{i}}{\partial p_{j}}=\frac{\partial x_{j}}{\partial w_{i}}
$$

This last relationship follows from the invariance of the Poisson bracket of $x_{j}$ and $J_{i}$ under a canonical transformation from the $x_{i}, p_{j}$ variables to the $w_{i}, J_{j}$ variables.

In the present article, we will be interested in only the equilibrium probability distribution $P\left(J_{1}, J_{2}, J_{3}\right)$ with no time dependence, and in situations involving radiation balance where there is no change in the radiation spectrum, and therefore no energy current within the mechanical system carrying energy from one frequency to another. In this case, the FokkerPlanck equation in Eq. (14) becomes the condition for each index $i$ that [9]

$$
0=-A_{i} P\left(J_{1}, J_{2}, J_{3}\right)+\sum_{j} B_{i j} \frac{\partial}{\partial J_{j}} P\left(J_{1}, J_{2}, J_{3}\right) .
$$

In 1980, Marshall and Claverie[9] applied their understanding of quasi-Markov processes to the classical hydrogen atom in classical electromagnetic zero-point radiation. Our equation (19) corresponds to their Eq. (5.2). They concluded that radiation balance did not exist for this system, but rather the scattering by the orbiting electron in the Coulomb potential was pushing the radiation spectrum away from the Lorentz-invariant zero-point form over toward some other spectrum. Later Claverie and Soto indicated that the hydrogen system was self-ionizing. [10]

\section{HYDROGEN SYSTEM}

\section{A. Sensitive Connection to the Random Radiation Spectrum}

Although in 1965, Marshall showed that the spectrum of classical electromagnetic zeropoint radiation was Lorentz invariant, [23] the physicists working with classical zero-point 
radiation in the 1960s and early 1970s did not appreciate the importance of using classical electromagnetic systems which are consistent with relativistic behavior. Here we note that the system of charged particles interacting through the Coulomb potential (such as the classical hydrogen atom) can be extended to full classical electrodynamics, and so provide a fully relativistic theory. Also, small linear-oscillator systems can be regarded as relativistic in the limit of small oscillation amplitude.

Most of the successes for mechanical systems coupled to zero-point radiation involve harmonic oscillator systems where the mechanical system has a fundamental mechanical frequency $\omega_{0}$ and interacts through the dipole approximation with radiation only at this same frequency $\omega_{0}$. On the other hand, the classical hydrogen atom has several properties which are in striking contrast with the properties of a harmonic oscillator. For example, the classical hydrogen atom has no natural mechanical oscillation frequency for the system. Indeed, a particle in a Coulomb potential allows all frequencies with no limits on frequency, $0<\omega<\infty$.

Furthermore, the harmonic oscillator and the hydrogen atom are very different in the appearance of time Fourier components associated with different frequencies of oscillation. The purely harmonic oscillator has an electric dipole moment at the natural frequency $\omega_{0}$ of the oscillator and at no other frequency. The mechanical motion is purely sinusoidal. Even for the case of a charged particle in an isotropic three-dimensional harmonic potential with natural frequency $\omega_{0}$ (where elliptical orbits appear), the small-amplitude motion is equivalent to three independent one-dimensional linear oscillations, each oscillation having an electric dipole moment oscillating at $\omega_{0}$ only; there are no dipole moments at any higher multiples of $\omega_{0}$. In complete contrast with this linear oscillator situation, a nonrelativistic elliptical electron orbit in a Coulomb potential with eccentricity $\epsilon>0$ has time Fourier components of velocity, not just at the frequency $\omega$ of the orbital motion, but at all multiples of $\omega$. Thus even in the most basic approximation, the dipole approximation, the elliptical nonrelativistic particle orbit (with the Coulomb source at one focus) is connected to the electromagnetic radiation at not just one frequency $\omega$ but at an infinite number of frequencies $n \omega$ for all integers $n$. Thus a charged particle in a Coulomb potential is a nonlinear mechanical system where the mechanical motion alone will determine the associated equilibrium spectrum of random radiation. The classical hydrogen atom is an enormously sensitive system in connection with radiation equilibrium since questions of radiation balance exist 
already in the dipole approximation.

We should emphasize that the one successful relativistic scattering calculation[15] involves entirely different aspects from these nonlinear systems. In complete contrast with nonlinear systems (such as nonlinear oscillators or hydrogen), the successful relativistic calculation involves a harmonic oscillator with just one time Fourier component of velocity at the one fundamental oscillator frequency $\omega_{0}$. The connection to the overtone frequencies is made, not through any time Fourier components at higher frequencies (which are nonexistent), but rather through the higher electric radiation multipole moments (beyond the electric dipole moment, such as quadrupole, etc.) which are associated with the finite amplitude of the oscillator motion and which connect the motion to the full space-and-time dependence of the radiation field $\mathbf{E}(\mathbf{r}, t)$. This relativistic calculation emphasizes the importance of relativity but gives no information about the hydrogen system.

For nonlinear oscillators treated in the dipole approximation, the Fokker-Planck analysis developed by Marshall and Claverie makes clear that it is the unperturbed mechanical motion which determines the spectrum of random radiation with which the oscillator is in equilibrium. The relativistic nature of electromagnetic waves does not appear in the dipole approximation which invokes $e \mathbf{E}(0, t)$ for the random force nor in the term in $\dddot{\mathbf{r}}$ for radiationdamping. Accordingly, any aspect of relativity must arise from the mechanical motion of the charged particle. For the classical hydrogen atom, it is the time Fourier components for the orbiting electron which will determine the spectrum of radiation equilibrium. However, only the time Fourier components for nonrelativistic elliptical particle motions in a Coulomb potential appear in the literature. Landau and Lifshitz give a calculation of the time Fourier components of a nonrelativistic particle moving in a Coulomb elliptical orbit, [24] and these dipole moments were then used in the radiation-balance analysis of Marshall and Claverie. Because the time Fourier components of the electromagnetic relativistic particle motion are different from those of the nonrelativistic elliptical orbital motion, the possibility arises that a treatment consistent with relativistic behavior may give rise to radiation balance whereas the completely nonrelativistic treatment does not. 


\section{B. Qualitative Suggestion for Equilibrium}

The classical hydrogen atom in classical zero-point radiation has attracted attention at least since 1975. In a review article published that year, it was suggested that there was hope that a classical hydrogen atom in zero-point radiation would have the same size as suggested by quantum theory. [5] It was noted[25] that the electron $e$ in a nonrelativistic circular orbit where $\omega=\left[e^{2} /\left(m r^{3}\right)\right]^{1 / 2}$ would emit radiation, and so lose nonrelativistic energy $\mathcal{E}$ at a rate

$$
\frac{d \mathcal{E}_{\text {loss }}}{d t}=\frac{2 e^{2}}{3 c^{3}} \omega^{4} r^{2}=\frac{2 e^{6}}{3 m^{2} c^{3} r^{4}} .
$$

while the nonrelativistic energy gain from zero-point radiation was estimated as

$$
\frac{d \mathcal{E}_{\text {gain }}}{d t}=\frac{e^{2} \hbar \omega^{3}}{2 m c^{3}}=\frac{e^{5} \hbar}{2 m^{5 / 2} c^{3} r^{9 / 2}}
$$

by using the calculation for a rotating electric dipole in zero-point radiation. Then energy balance required that $m \omega r^{2}=(3 / 4) \hbar$ which (except for the unreliable factor of $3 / 4$ ) agrees with Bohr's condition $m \omega r^{2}=\hbar$ for the hydrogen ground state in quantum theory. In this qualitative analysis, there is no suggestion that relativistic behavior for the particle motion may play a role in the equilibrium situation.

\section{Action-Angle Variables for Hydrogen}

The action-angle variables for a particle in a Coulomb potential have been given in several books. The action-angle variables for nonrelativistic hydrogen appear in Goldstein's textbook of classical mechanics; [26] the action-angle variables for both nonrelativistic and relativistic hydrogen are given in Born's monograph, Mechanics of the Atom. [17] Only the relativistic situation corresponds to electrodynamics. The component of relativistic angular

momentum along the $z$-axis is $J_{3}=J_{\phi}=m_{0} \gamma(r \sin \theta)^{2} \dot{\phi}$ where $\gamma=\left(1-v^{2} / c^{2}\right)^{-1 / 2}$, and the magnitude of the total angular momentum is denote as $J_{2}, J_{2}=J_{\theta}+J_{\phi}$. The relativistic energy $U\left(J_{r}, J_{\theta}, J_{\phi}\right)$ (including the rest energy $\left.m_{0} c^{2}\right)$ is

$$
U\left(J_{r}, J_{\theta}, J_{\phi}\right)=m_{0} c^{2}\left(1+\left[\frac{e^{2} / c}{J_{r}+\sqrt{\left(J_{\theta}+J_{\phi}\right)^{2}-\left(e^{2} / c\right)^{2}}}\right]^{2}\right)^{-1 / 2} .
$$

By taking derivatives of the Hamiltonian with respect to the action variables, we can obtain the corresponding relativistic (angular) frequencies 


$$
\omega_{r}=\frac{m_{0} c^{2}\left(e^{2} / c\right)^{2}}{\left[\left(e^{2} / c\right)^{2}+\left(J_{r}+\sqrt{J_{2}^{2}-\left(e^{2} / c\right)^{2}}\right)^{2}\right]^{3 / 2}}
$$

and

$$
\omega_{\theta}=\omega_{\phi}=\omega_{2}=\frac{m_{0} c^{2}\left(e^{2} / c\right)^{2}}{\sqrt{1-\left[e^{2} /\left(J_{2} c\right)\right]^{2}}\left[\left(e^{2} / c\right)^{2}+\left(J_{r}+\sqrt{J_{2}^{2}-\left(e^{2} / c\right)^{2}}\right)^{2}\right]^{3 / 2}} .
$$

We note that the relativistic expressions for $\omega_{r}$ and $\omega_{2}$ are not equal, rather $\omega_{r}=\Gamma \omega_{2}$ where there is a relative factor of

$$
\Gamma=\sqrt{1-\left[e^{2} /\left(J_{2} c\right)\right]^{2}}
$$

The relativistic orbital equation for a particle in a Coulomb potential is

$$
r=\frac{a\left(1-\epsilon^{2}\right)}{1+\epsilon \cos (\Gamma \phi)}
$$

where the length $a$ depends only upon the energy $U$

$$
a=\frac{e^{2} U}{\left(m_{0} c^{2}\right)^{2}-U^{2}}
$$

and not upon the eccentricity $\epsilon$

$$
\epsilon=\sqrt{1-\frac{1}{\left[1+J_{r} / \sqrt{J_{2}^{2}-\left(e^{2} / c\right)^{2}}\right]^{2}}}=\sqrt{1-\frac{1}{\left[1+J_{r} /\left(J_{2} \Gamma\right)\right]^{2}}} .
$$

The connection between the angle $\phi(t)$ and the time $t$ depends on the implicit relationship

$$
t=\int_{0}^{\phi} d \phi^{\prime} \frac{r^{2}\left[U+e^{2} / r\right]}{c^{2} J_{2}}=\left[1+\frac{\left(1-\epsilon^{2}\right)}{\left[\left(m_{0} c^{2} / U\right)^{2}-1\right]}\right]^{-1 / 2} \int_{0}^{\phi} \frac{d \phi^{\prime}}{c}\left(\frac{r^{2} U}{e^{2}}+r\right)
$$

\section{The Need to Compare the Relativistic and Nonrelativistic Calculations}

All the calculation to date on the classical hydrogen atom in zero-point radiation have been made in the nonrelativistic approximation. Thus the suggestive qualitative calculation above[5] in Eqs. (20) and (21), the work of Marshall and Claverie, [9] and the simulations of Cole and Zou[11] have all started with a nonrelativistic charged particle in a Coulomb potential. However, it was emphasized in 2004 that relativistic Coulomb orbits can be qualitatively 
different from the familiar conic-section orbits of nonrelativistic physics.[27] Knowledge of this contrast between the nonrelativistic and relativistic orbits, taken together with the new relativistic scattering calculation, [15] emphasizes that relativity may be important for the classical hydrogen atom. The nonrelativistic limit corresponds to $e^{2} /\left(J_{2} c\right)<<1$. The nonrelativistic limits of all the quantities in Eqs. (22) through (28) are obtained in this large- $J_{2}$ limit.

\section{E. Multiply-Periodic Relativistic Coulomb Orbits}

In the dipole approximation, radiation balance depends upon the time Fourier components of the electric dipole moment calculated from the particle's unperturbed orbital motion. The dipole moment for the classical charged particle in a Coulomb potential is obtained by multiplying the charge $e$ times the vector displacement, $e \mathbf{r}(t)=\widehat{e i x}(t)+\widehat{j j} y(t)$. Now for a multiply-periodic system involving a central potential, such as the relativistic classical hydrogen atom, the $x$ - and $y$-components of planar orbits can be expanded in the form

$$
\begin{aligned}
x(t) & =\sum_{k} D_{k}\left(J_{r}, J_{2}\right) \exp \left[i k w_{r}+i w_{2}\right] \\
& =\sum_{k} D_{k}\left(J_{r}, J_{2}\right) \exp \left[i k\left(\omega_{r} t+\beta_{r}\right)+i\left(\omega_{2} t+\beta 2\right)\right] \\
& =\sum_{k} x_{k}\left(J_{r}, J_{2}\right) \exp \left[i(1+k \Gamma) \omega_{2} t\right]
\end{aligned}
$$

where (up to a factor of $e$ ) the coefficients $x_{k}\left(J_{r}, J_{2}\right)$ will provide the time Fourier components of the electric dipole moment oscillating with angular frequency $\omega_{2}+k \omega_{r}=(1+k \Gamma) \omega_{2}$.

\section{F. Periodic Nonrelativistic Coulomb Orbits}

Although the particle motion of a charged particle in a Coulomb potential in electromagnetism is multiply periodic, the motion of a nonrelativistic particle in a Coulomb potential is periodic. In contrast with the time Fourier components of the relativistic orbital motion $x_{k}$

given in Eq. (29), the time Fourier components $x_{n}^{N R}$ of the nonrelativistic elliptical orbital 
motion of the electron in the Coulomb potential appear in the expansion

$$
\begin{aligned}
x_{N R}(t) & =\sum_{k} D_{N R k}\left(J_{r}, J_{2}\right) \exp \left[i k w_{r}+i w_{2}\right] \\
& =\sum_{k} D_{N R k}\left(J_{r}, J_{2}\right) \exp \left[i k\left(\omega_{N R} t+\beta_{r}\right)+i\left(\omega_{N R} t+\beta_{2}\right)\right] \\
& =\sum_{n} x_{n}^{N R} \exp \left[i n \omega_{N R} t\right]
\end{aligned}
$$

since in the nonrelativistic limit there is only one frequency involved, $\omega_{N R}=\omega_{r}=\omega_{2}$. In the nonrelativistic limit (which sends $\Gamma \rightarrow 1$ since $c \rightarrow \infty$ ), each term in Eq. (29) makes a contribution to one of the terms in Eq. (30); however, a single term in Eq. (30) may receive contributions from more than one term in Eq. (29).

\section{G. Descriptions of Hydrogen Require Time Averaging}

Attempts to understand both the stability and radiation balance for the hydrogen atom involve calculations over extended periods of time. Numerical simulations follow the electron motion over many orbits, and the generalized Fokker-Planck analysis (sketched above) requires time averaging over the unperturbed particle motion in order to compute the drift and diffusion coefficients. However, there is a crucial contrast between the relativistic and nonrelativistic particle motion in a hydrogen atom. The relativistic motion involves a rosette motion of a rotating elliptical orbit whereas the nonrelativistic motion involves an elliptical orbit which is fixed in orientation and is not rotating. On account of this contrast in fundamental orbital motion, the relativistic and nonrelativistic time Fourier expansions are different, and calculational results may depend upon whether the orbital motion over an extended time is evaluated before or after taking the nonrelativistic limit.

If we first average over the rosette motion of the relativistic orbit, then the time averages of $x^{2}$ and $y^{2}$ are identical, and when now taking the nonrelativistic limit, this agreement between the time averages of $x^{2}$ and $y^{2}$ will persist. On the other hand, if we first take the nonrelativistic limit for the particle motion, then the relativistic rosette motion reduces to a nonrelativistic non-rotating ellipse whose orientation in space is fixed. Let's assume (following Landau and Lifshitz) that the major axis of the ellipse is along the $x$-axis and the minor axis along the $y$-axis. Then for any ellipse with non-zero eccentricity $\epsilon>0$, the time average value of $x^{2}$ will be different from the time average of $y^{2}$, since the squares of the semimajor and semiminor axes differ as $a^{2}$ and $a^{2}\left(1-\epsilon^{2}\right)$. As the eccentricity $\epsilon$ becomes 
larger, the difference between the time averages for $x^{2}$ and $y^{2}$ will become larger, and hence the departure from the relativistic situation becomes larger.

The relativistic distinction between $\omega_{r}$ and $\omega_{2}$, which leads to rosette motion, may well be important in connection with numerical simulations involving energy absorption from random radiation. In the nonrelativistic approximation which equates $\omega_{r}$ and $\omega_{2}$, the unperturbed motion follows the same spatial path repeatedly, and so the particle can pick up significant energy from the higher harmonics. On the other hand, in the relativistic description, the spatial path is not repeated but rather shifts in orientation and velocity, and so the particle is less likely to pick up energy from the higher radiation harmonics which involve higher frequencies and smaller wavelengths. This distinction may be important in the numerical calculations (such as those of Nieuwenhuizen and Liska[12][14] where selfionization is found in orbits of high eccentricity.

\section{H. Dipole Approximation, Eccentricity, and Radiation Balance}

The use of the dipole approximation for the radiation emission term and the radiationforcing term in Eq. (13) implies that the radiation speed is vastly larger than any particle speed; there is no actual comparison between the two. In the first Bohr orbit of old quantum theory, the electron speed is nonrelativistic, and it is usually assumed that nonrelativistic approximations are valid in any treatment of the hydrogen atom. However, the comments above regarding the time Fourier coefficients of the relativistic versus nonrelativistic orbital motions must make us cautious.

The use of the dipole approximation for the interaction between radiation and matter means that radiation balance depends entirely upon the time Fourier components of the unperturbed particle motion, and therefore upon the eccentricity $\epsilon$. If the orbit is circular, $\epsilon=0$, then there is exactly one coefficient $x_{1}\left(J_{2}\right)$ at frequency $\omega_{2}$ in the time Fourier expansion for the particle motion $x(t)$ in Eq. (29). In this case, the mechanical motion interacts with the radiation at this one frequency and at no other frequency; at a single time $t$, there is no transfer of energy between radiation at two different frequencies. However, if the eccentricity of the particle orbit is non-zero, $\epsilon>0$, then there are nonvanishing coefficients $x_{k}\left(J_{r}, J_{2}\right)$ at several frequencies, and, at a single time $t$, the orbital motion of the charged particle can indeed transfer energy among different radiation frequencies. For example, 
through first order in $\epsilon$, the orbital motion has time Fourier components at frequencies $\omega_{2}+\omega_{r}$ and $\omega_{2}-\omega_{r}$, and so, at a single time $t$, the orbital motion can transfer energy between these two radiation frequencies. As the eccentricity $\epsilon$ increases toward $\epsilon \rightarrow 1$, more and more time Fourier coefficients $x_{k}\left(J_{r}, J_{2}\right)$ will become important, and the mechanical motion can transfer energy among many radiation frequencies. However, we will limit out attention to the simplest case. The moment that more than one frequency is involved, it is possible to test for radiation balance because the mechanical system can transfer energy between the radiation modes at these differing frequencies. Thus we will limit our calculations to particle motions which are nearly circular and where the eccentricity $\epsilon$ is small. Our calculations will hold through first order in $\epsilon$ for the time-Fourier-coefficient expansions for $x(t)$ and $y(t)$, and through order $\epsilon^{2}$ for the squares of the particle coordinates.

\section{Failure of the General Calculation in Marshall and Claverie's Analysis}

The limitation of our calculations to only first-order-in- $\epsilon$ departures from circular motion corresponds to the situation where the nonrelativistic approximation is consistent with the accurate relativistic analysis. We will not consider the enormously complex general problem of radiation equilibrium at higher orders in $\epsilon$. Indeed, if we examine equations (5.24) and (5.25) in Marshall and Claverie's work but stop at the lowest order in $\epsilon$, then their criterion for radiation balance is satisfied. However, Marshall and Claverie regarded their calculations as valid to all orders in $\epsilon$, and such regard is erroneous.

The failure of Marshall and Claverie's calculations goes back to a failure in Landau and Lifshitz's Classical Theory of Fields. [24] The mathematics in the textbook is not in question. Rather, the error is in the physical assumptions. Landau and Lifshitz assume that the nonrelativistic limits of the time Fourier components of the relativistic particle motion are the same as the time Fourier components of the nonrelativistic approximation to the orbital motion. Landau and Lifshitz assume that the multiply periodic expansion for a relativistic charged particle motion in a Coulomb potential given in our Eq. (29) can be represented in the nonrelativistic limit by the periodic expansion in Eq. (30). Such assumptions are not valid for the electrodynamics of a charge particle in a Coulomb potential where the motion is necessarily relativistic. Thus the time Fourier components $x_{k}, y_{k}$ of the relativistic motion are equal except for a relative phase corresponding to changing a cosine 
time dependence into a sinusoidal time dependence. However, in the periodic nonrelativistic

elliptical motion assumed by Landau and Lifshitz, the time Fourier components $x_{n}^{N R}, y_{n}^{N R}$ are quite different corresponding to expanding the semimajor and semiminor axes of an ellipse. In their calculations, Landau and Lifshitz assume that the semimajor axis of the stationary nonrelativistic ellipse is along the $x$-axis, and give the time Fourier expansion coefficients following from (301) in terms of Bessel functions $J_{n}(n \epsilon)$ and derivatives $J_{n}^{\prime}(n \epsilon)$, as

$$
x_{n}^{N R}=\frac{a}{n} J_{n}^{\prime}(n \epsilon)=\frac{a}{2}\left(\frac{n \epsilon}{2}\right)^{n-1}+\ldots
$$

and

$$
y_{n}^{N R}=\frac{a \sqrt{1-\epsilon^{2}}}{n \epsilon} J_{n}(n \epsilon)=\frac{a \sqrt{1-\epsilon^{2}}}{2}\left(\frac{n \epsilon}{2}\right)^{n-1}+\ldots
$$

Here, as in the later parts of Marshall and Claverie's work, the index $n$ follows the notation of Landau and Lifshitz rather that the earlier summation notation given as $k$ in Eq. (30). These time Fourier expansion coefficients start with $n=1$. Use of Landau and Lifshitz's expressions for $x_{n}^{N R}$ and $y_{n}^{N R}$ through first order in $\epsilon$ leads to the same results which we will present below. Indeed, only the first two terms (corresponding to $n=1$ and $n=2$ ) are given accurately through first order in $\epsilon$. In order $\epsilon^{2}$ and beyond, there is disagreement with electrodynamics because of questions of the order of time Fourier evaluations and nonrelativistic limits.

\section{RADIATION BALANCE FOR HYDROGEN ORBITS THROUGH FIRST OR- DER IN ECCENTRICITY}

\section{A. Calculation of Time Fourier Components Through First Order in Eccentricity}

In the present article, we will consider the question of radiation balance only through first order in the orbital eccentricity. We need to substitute the relativistic orbital relations into

$$
\begin{aligned}
& x(t)=r[\phi(t)] \cos \phi(t), \\
& y(t)=r[\phi(t)] \sin \phi(t) .
\end{aligned}
$$


Expanding the expression (25) for $r(\phi)$ through first order in $\epsilon$ gives

$$
r=a-\epsilon a \cos \Gamma \phi
$$

Squaring this expression gives through order $\epsilon$,

$$
r^{2}=a^{2}-\epsilon 2 a^{2} \cos \Gamma \phi
$$

Substituting Eq. (35) into Eqs. (33) and (34) and using trigonometric identities, we have

$$
x=r \cos \phi=a \cos \phi-\frac{\epsilon a}{2}\{\cos [(1+\Gamma) \phi]+\cos [(1-\Gamma) \phi]\}
$$

and

$$
y=r \sin \phi=a \sin \phi-\frac{\epsilon a}{2}\{\sin [(1+\Gamma) \phi]+\sin [(1-\Gamma) \phi]\} .
$$

We now need $\phi(t)$ through first order in $\epsilon$. We use Eq. (28) to obtain

$$
\begin{aligned}
t & =\int_{0}^{\phi} d \phi^{\prime} \frac{r^{2}\left[U+e^{2} / r\right]}{c^{2} J_{2}}=\frac{U}{c^{2} J_{2}} \int_{0}^{\phi} d \phi^{\prime} r^{2}+\frac{e^{2}}{c^{2} J_{2}} \int_{0}^{\phi} d \phi^{\prime} r \\
& =\frac{U}{c^{2} J_{2}} \int_{0}^{\phi} d \phi^{\prime}\left\{a^{2}-\epsilon 2 a^{2} \cos \Gamma \phi\right\}+\frac{e^{2}}{c^{2} J_{2}} \int_{0}^{\phi} d \phi^{\prime}\{a-\epsilon a \cos \Gamma \phi\} \\
& =\left(\frac{U}{c^{2} J_{2}} a^{2}+\frac{e^{2}}{c^{2} J_{2}} a\right) \phi-\epsilon\left(\frac{2 a^{2} U}{c^{2} J_{2} \Gamma}+\frac{e^{2} a}{c^{2} J_{2} \Gamma}\right) \sin \Gamma \phi
\end{aligned}
$$

We recognize

$$
\frac{1}{\omega_{2}}=\frac{a}{c^{2} J_{2}}\left(a U+e^{2}\right)
$$

and, for simplicity, we define

$$
F=\frac{2 a U+e^{2}}{a U+e^{2}}=2-\left(\frac{e^{2}}{J_{2} c}\right)^{2}
$$

Then Eq. (39) becomes

$$
\omega_{2} t=\phi-\epsilon \frac{F}{\Gamma} \sin \Gamma \phi
$$

and, through first order in $\epsilon$,

$$
\phi=\omega_{2} t+\epsilon \frac{F}{\Gamma} \sin \omega_{2} t
$$

Substituting this result for $\phi(t)$ into Eqs. (37) and (38), we find through first order in $\epsilon$

$$
x=a \cos \omega_{2} t+\epsilon a\left(-\frac{1}{2}+\frac{F}{2 \Gamma}\right) \cos \left[(1+\Gamma) \omega_{2} t\right]-\epsilon a\left(\frac{1}{2}+\frac{F}{2 \Gamma}\right) \cos \left[(1-\Gamma) \omega_{2} t\right]
$$


and

$$
y=a \sin \omega_{2} t+\epsilon a\left(-\frac{1}{2}+\frac{F}{2 \Gamma}\right) \sin \left[(1+\Gamma) \omega_{2} t\right]-\epsilon a\left(\frac{1}{2}+\frac{F}{2 \Gamma}\right) \sin \left[(1-\Gamma) \omega_{2} t\right] .
$$

Here we have the time Fourier expansion through first order in $\epsilon$ for the relativistic particle motion. Note that for these expressions which assume relativistic motion, we have exactly the same coefficients for the corresponding terms in $x$ and in $y$, which involve frequencies $\omega_{2}, \omega_{2} \pm \omega_{r}=(1 \pm \Gamma) \omega_{2}$.

If we take the nonrelativistic limit of these expression, then $\Gamma \rightarrow 1$ and $F \rightarrow 2$, and we notice a contrast between the expressions for $x_{N R}(t)$ and $y_{N R}(t)$ arising from $\cos \left[(1-\Gamma) \omega_{2} t\right] \rightarrow \cos 0=1$ and $\sin \left[(1-\Gamma) \omega_{2} t\right] \rightarrow \sin 0=0$. Thus the nonrelativistic limits of the time Fourier expansions for the relativistic particle motion become

$$
x \rightarrow x_{N R}=-\frac{3 \epsilon a}{2}+a \cos \omega_{N R} t+\frac{\epsilon a}{2} \cos \left[2 \omega_{N R} t\right]
$$

and

$$
y \rightarrow y_{N R}=a \sin \omega_{N R} t+\frac{\epsilon a}{2} \sin 2 \omega_{N R} t .
$$

These expressions give the time Fourier components through first order in $\epsilon$ for $x_{N R}(t)$ and $y_{N R}(t)$ at frequencies $\omega_{N R}$ and $2 \omega_{N R}$.

Next we note the difference between the squares of the components depending upon the orders of time averaging and nonrelativistic limit. If we time average the squares of the relativistic expressions in Eqs. (44) and (45), and subsequently take the nonrelativistic limit, we have equal contributions for $\left\langle x^{2}\right\rangle$ and $\left\langle y^{2}\right\rangle$,

$$
\begin{aligned}
\left\langle x^{2}\right\rangle & =\left\langle y^{2}\right\rangle=\frac{1}{2}\left[a^{2}+\epsilon^{2} a^{2}\left(-\frac{1}{2}+\frac{F}{2 \Gamma}\right)^{2}+\epsilon^{2} a^{2}\left(\frac{1}{2}+\frac{F}{2 \Gamma}\right)^{2}\right] \\
& \rightarrow \frac{1}{2}\left[a^{2}+\epsilon^{2} a^{2}\left(\frac{1}{2}\right)^{2}+\epsilon^{2} a^{2}\left(\frac{3}{2}\right)^{2}\right]=\frac{a^{2}}{2}+\frac{5}{4} \epsilon^{2} a^{2} .
\end{aligned}
$$

On the other hand, if the nonrelativistic limit is taken as in Eqs. (46) and (47) before the time averaging, we find that $\left\langle x_{N R}^{2}\right\rangle$ and $\left\langle y_{N R}^{2}\right\rangle$ are quite different,

$$
\left\langle x_{N R}^{2}\right\rangle=\left(\frac{3 \epsilon a}{2}\right)^{2}+\frac{1}{2}\left[a^{2}+\left(\frac{\epsilon a}{2}\right)^{2}\right]=\frac{a^{2}}{2}+\frac{19}{8} \epsilon^{2} a^{2}
$$

and

$$
\left\langle y_{N R}^{2}\right\rangle=\frac{1}{2}\left[a^{2}+\left(\frac{\epsilon a}{2}\right)^{2}\right]=\frac{a^{2}}{2}+\frac{1}{8} \epsilon^{2} a^{2} .
$$


When dealing with higher-order terms in the eccentricity $\epsilon$, the nonrelativistic limit will involve terms beyond the simple relative constant of $-3 \epsilon a / 2$ which appears between Eqs. (46) and (47). For example, terms in $\epsilon^{2}$ in $x$ and $y$ will introduce frequencies $(1+2 \Gamma) \omega_{2}$ and $(1-2 \Gamma) \omega_{2}$. In the nonrelativistic limits, these frequencies become $3 \omega_{N R}$ and $\omega_{N R}$, so that the coefficients of the terms in $\cos \omega_{N R} t$ and $\sin \omega_{N R} t$ will be altered. With higher order terms in the nonrelativistic approximation, the time Fourier expansions for $x$ and $y$ become increasingly different, and the departure from valid electrodynamics increases.

Landau and Lifshitz give the time Fourier coefficients for the velocity of a nonrelativistic particle in a Coulomb potential, and so they do not include the constant term which appears in our nonrelativistic limit for $x$ given in Eq. (46). Because Landau and Lifshitz take the limit over to nonrelativistic orbital motion before obtaining the time Fourier coefficients of the particle motion, their time Fourier coefficients correspond to electromagnetism only through first order in $\epsilon$.

\section{B. Obtaining the Fokker-Planck Equations}

From the time Fourier expansions in Eqs. (44) and (45), it is clear that for the relativistic particle motion through order $\epsilon$, we will have time Fourier components at frequencies $\omega_{2}$, $(1+\Gamma) \omega_{2}$, and $(1-\Gamma) \omega_{2}$. Thus the relativistic electron in the Coulomb potential can transfer energies among these frequencies.

In order to take advantage of Marshall and Claverie's analysis, [9] we will rewrite Eqs. (44) and (45) in terms of the angle variables $w_{r}=\omega_{r} t+\beta_{r}$ and $w_{2}=\omega_{2} t+\beta_{2}$. We have through terms first order in $\epsilon$

$$
x(t)=a \cos w_{2}+\epsilon a\left[G \cos \left(w_{2}+w_{r}\right)-H \cos \left(w_{2}-w_{r}\right)\right],
$$

and

$$
y(t)=a \sin w_{2}+\epsilon a\left[G \sin \left(w_{2}+w_{r}\right)-H \sin \left(w_{2}-w_{r}\right)\right]
$$

where

$$
G=\frac{1}{2 \Gamma}(-\Gamma+F)=\frac{1}{2 \Gamma}\left(-\Gamma+\frac{2 a U+e^{2}}{a U+e^{2}}\right)
$$

and

$$
H=\frac{1}{2 \Gamma}(\Gamma+F)=\frac{1}{2 \Gamma}\left(\Gamma+\frac{2 a U+e^{2}}{a U+e^{2}}\right) .
$$


Now we want to evaluate Marshall and Claverie's quantities $A_{r}, A_{2}, B_{r r}, B_{r 2}, B_{22}$. We will need the quantity following from Eq. (18)

$$
\left\langle\dddot{x}(t) \frac{\partial J_{r}}{\partial p_{x}}+\dddot{y}(t) \frac{\partial J_{r}}{\partial p_{y}}\right\rangle=\left\langle\dddot{x}(t) \frac{\partial x}{\partial w_{r}}+\dddot{y}(t) \frac{\partial y}{\partial w_{r}}\right\rangle .
$$

We then evaluate $\dddot{x}(t)$ and $\partial x / \partial w_{r}$ from Eq. (51), and $\dddot{y}(t)$ and $\partial y / \partial w_{r}$ from Eq. (52), giving

$$
\begin{aligned}
& \left\langle\dddot{x}(t) \frac{\partial x}{\partial w_{r}}\right\rangle \\
& =\left\langle\left[a \omega_{2}^{3} \sin w_{2}+\epsilon a\left[G\left(\omega_{2}+\omega_{r}\right)^{3} \sin \left(w_{2}+w_{r}\right)+H\left(\omega_{2}-\omega_{r}\right)^{3} \sin \left(w_{2}-w_{r}\right)\right]\right]\right. \\
& \left.\times\left\{0+\epsilon a\left[-G \sin \left(w_{2}+w_{r}\right)-H \sin \left(w_{2}-w_{r}\right)\right]\right\}\right\rangle
\end{aligned}
$$

since $\partial w_{r} / \partial t=\omega_{r}$ and $\partial w_{2} / \partial t=\omega_{2}$, and there is a similar term in $\left\langle\dddot{y} \partial y / \partial w_{r}\right\rangle$. Here on time averaging, we have $w_{r}=\omega_{r} t+\beta_{r}$ and $w_{2}=\omega_{2} t+\beta_{2}$ so that

$$
\left\langle\sin ^{2} w\right\rangle=\left\langle\cos ^{2} w\right\rangle=1 / 2
$$

while

$$
\langle\sin w \cos w\rangle=0 \text {. }
$$

Then combining Eqs. (55) and (56), and the analogous terms in $y$, we have

$$
\begin{aligned}
A_{r} & =\frac{2 e^{2}}{3 c^{3}}\left\langle\dddot{x}(t) \frac{\partial J_{r}}{\partial p_{x}}+\dddot{y}(t) \frac{\partial J_{r}}{\partial p_{y}}\right\rangle=\frac{2 e^{2}}{3 c^{3}}\left\langle\dddot{x}(t) \frac{\partial x}{\partial w_{r}}+\dddot{y}(t) \frac{\partial y}{\partial w_{r}}\right\rangle \\
& =-\frac{2 e^{2}}{3 c^{3}} \frac{\epsilon^{2} a^{2}}{2}\left[G^{2}\left(\omega_{2}+\omega_{r}\right)^{3}+H^{2}\left(\omega_{2}-\omega_{r}\right)^{3}\right] .
\end{aligned}
$$

Proceeding in a similar fashion, we obtain

$$
\begin{aligned}
A_{2} & =\frac{2 e^{2}}{3 c^{3}}\left\langle\dddot{x}(t) \frac{\partial J_{2}}{\partial p_{x}}+\dddot{y}(t) \frac{\partial J_{2}}{\partial p_{y}}\right\rangle=\frac{2 e^{2}}{3 c^{3}}\left\langle\dddot{x}(t) \frac{\partial x}{\partial w_{2}}+\dddot{y}(t) \frac{\partial y}{\partial w_{2}}\right\rangle \\
& =-\frac{2 e^{2}}{3 c^{3}}\left\{a^{2} \omega_{2}^{3}+\frac{\epsilon^{2} a^{2}}{2}\left[G^{2}\left(\omega_{2}+\omega_{r}\right)^{3}+H^{2}\left(\omega_{2}-\omega_{r}\right)^{3}\right]\right\} .
\end{aligned}
$$

Next we need averages over the time $t$ involving

$$
\left\langle\frac{\partial J_{r}}{\partial p_{x}}(t) \frac{\partial J_{r}}{\partial p_{x}}(t+\tau)+\frac{\partial J_{r}}{\partial p_{y}}(t) \frac{\partial J_{r}}{\partial p_{y}}(t+\tau)\right\rangle .
$$


The term in $x$ involves

$$
\begin{aligned}
& \left\langle\frac{\partial J_{r}}{\partial p_{x}}(t) \frac{\partial J_{r}}{\partial p_{x}}(t+\tau)\right\rangle=\left\langle\frac{\partial x}{\partial w_{r}}(t) \frac{\partial x}{\partial w_{r}}(t+\tau)\right\rangle \\
& =\left\langle\left\{0+\epsilon a\left[-G \sin \left(w_{2}+w_{r}\right)-H \sin \left(w_{2}-w_{r}\right)\right]\right\}\right. \\
& \left.\times\left\{0+\epsilon a\left[-G \sin \left(w_{2}+w_{r}+\omega_{2} \tau+\omega_{r} \tau\right)-H \sin \left(w_{2}-w_{r}+\omega_{2} \tau-\omega_{r} \tau\right)\right]\right\}\right\rangle \\
& =\frac{\epsilon^{2} a^{2}}{2}\left[G^{2} \cos \left(\omega_{2} \tau+\omega_{r} \tau\right)+H^{2} \cos \left(\omega_{2} \tau-\omega_{r} \tau\right)\right]
\end{aligned}
$$

with an analogous term in $y(t)$. Then we find

$$
\begin{aligned}
B_{r r} & =\frac{e^{2}}{2} \int_{-\infty}^{\infty} d \tau B_{E}(\tau)\left\langle\frac{\partial J_{r}}{\partial p_{x}}(t) \frac{\partial J_{r}}{\partial p_{x}}(t+\tau)+\frac{\partial J_{r}}{\partial p_{y}}(t) \frac{\partial J_{r}}{\partial p_{y}}(t+\tau)\right\rangle \\
& =\frac{e^{2}}{2} \epsilon^{2} a^{2} \int_{-\infty}^{\infty} d \tau B_{E}(\tau)\left[G^{2} \cos \left(\omega_{2} \tau+\omega_{r} \tau\right)+H^{2} \cos \left(\omega_{2} \tau-\omega_{r} \tau\right)\right] \\
& =\epsilon^{2} a^{2} \frac{2 e^{2}}{3 c^{3}} \frac{\hbar}{2}\left\{G^{2}\left(\omega_{2}+\omega_{r}\right)^{3}+H^{2}\left(\omega_{2}-\omega_{r}\right)^{3}\right\}
\end{aligned}
$$

where we evaluated the time integrals as in Eqs. (6) and (7) and have used $S_{\text {Ezp }}(\omega)$ as given in Eq. (7). In a similar fashion, we obtain

$$
\begin{aligned}
B_{r 2} & =\frac{e^{2}}{2} \int_{-\infty}^{\infty} d \tau B_{E}(\tau)\left\langle\frac{\partial J_{r}}{\partial p_{x}}(t) \frac{\partial J_{2}}{\partial p_{x}}(t+\tau)+\frac{\partial J_{r}}{\partial p_{y}}(t) \frac{\partial J_{2}}{\partial p_{y}}(t+\tau)\right\rangle \\
& =\frac{e^{2}}{2} \epsilon^{2} a^{2} \int_{-\infty}^{\infty} d \tau B_{E}(\tau)\left[G^{2} \cos \left(\omega_{2} \tau+\omega_{r} \tau\right)+H^{2} \cos \left(\omega_{2} \tau-\omega_{r} \tau\right)\right] \\
& =\epsilon^{2} a^{2} \frac{2 e^{2}}{3 c^{3}} \frac{\hbar}{2}\left\{G^{2}\left(\omega_{2}+\omega_{r}\right)^{3}+H^{2}\left(\omega_{2}-\omega_{r}\right)^{3}\right\}
\end{aligned}
$$

and

$$
\begin{aligned}
B_{22} & =\frac{e^{2}}{2} \int_{-\infty}^{\infty} d \tau B_{E}(\tau)\left\langle\frac{\partial J_{2}}{\partial p_{x}}(t) \frac{\partial J_{2}}{\partial p_{x}}(t+\tau)+\frac{\partial J_{2}}{\partial p_{y}}(t) \frac{\partial J_{2}}{\partial p_{y}}(t+\tau)\right\rangle \\
& =\frac{e^{2}}{2}\left\{a^{2} \int_{-\infty}^{\infty} d \tau B_{E}(\tau) \cos \omega_{2} \tau\right. \\
& \left.+\epsilon^{2} a^{2} \int_{-\infty}^{\infty} d \tau B_{E}(\tau)\left[G^{2} \cos \left(\omega_{2} \tau+\omega_{r} \tau\right)+H^{2} \cos \left(\omega_{2} \tau-\omega_{r} \tau\right)\right]\right\} \\
& =a^{2} \frac{2 e^{2}}{3 c^{3}} \frac{\hbar}{2} \omega_{2}^{3}+\epsilon^{2} a^{2} \frac{2 e^{2}}{3 c^{3}} \frac{\hbar}{2}\left[G^{2}\left(\omega_{2}+\omega_{r}\right)^{3}+H^{2}\left(\omega_{2}-\omega_{r}\right)^{3}\right]
\end{aligned}
$$

\section{Using the Fokker-Planck Equations Associated with Radiation Balance}

\section{Circular Orbits}

In the case of circular motion where the eccentricity vanishes, $\epsilon=0$, the particle motion is connected to only one frequency, the fundamental frequency $\omega_{2}$. In this case, radiation 
balance always holds since at any time $t$ energy can be exchanged with only one frequency. In this circular case, the action variable $J_{r}$ vanishes, and Marshall and Claverie's coefficients simplify to $A_{r}=0, B_{r r}=0, B_{r 2}=0$,

$$
A_{2}=-\frac{2 e^{2}}{3 c^{3}}\left\{a^{2} \omega_{2}^{3}\right\}, \text { circular case. }
$$

and

$$
B_{22}=\frac{2 e^{2}}{3 c^{3}} \frac{\hbar}{2}\left\{a^{2} \omega_{2}^{3}\right\}, \text { circular case. }
$$

Then the non-vanishing equation for equilibrium requires

$$
\begin{aligned}
0 & =-A_{2} P\left(J_{2}\right)+B_{22} \frac{d P\left(J_{2}\right)}{d J_{2}} \\
& =\frac{2 e^{2}}{3 c^{3}}\left\{a^{2} \omega_{2}^{3}\right\} P\left(J_{2}\right)+\frac{2 e^{2}}{3 c^{3}} \frac{\hbar}{2}\left\{a^{2} \omega_{2}^{3}\right\} \frac{d P\left(J_{2}\right)}{d J_{2}}
\end{aligned}
$$

with the solution

$$
P\left(J_{2}\right)=\text { const } \times \exp \left[-\frac{J_{2}}{\hbar / 2}\right] .
$$

The solution suggests that the average value of the action variable $J_{2}$ of the mechanical Coulomb system is directly connected to Planck's constant $\hbar$ appearing as the multiplicative scale for zero-point radiation.

\section{Orbits of Small Eccentricity}

For the situation of circular orbits, there is no question about radiation balance, since the phase space distribution assures that the particle is in equilibrium with the radiation at the single frequency $\omega_{2}$. The crucial question is the radiation balance when the non-zero eccentricity of the orbit connects quantities at different frequencies such as $\omega_{2}, \omega_{2}+\omega_{r}$, and $\omega_{2}-\omega_{r}$.

Now using the full drift and diffusion coefficients in Eq. (59)-(64), the first Fokker-Planck equation, labeled by the index $r$, requires

$$
\begin{aligned}
0 & =-A_{r} P\left(J_{r}, J_{2}\right)+B_{r r} \frac{d P\left(J_{r}, J_{2}\right)}{d J_{r}}+B_{r 2} \frac{d P\left(J_{r}, J_{2}\right)}{d J_{2}} \\
& =\frac{2 e^{2}}{3 c^{3}} \epsilon^{2} a^{2}\left[G^{2}\left(\omega_{2}+\omega_{r}\right)^{3}+H^{2}\left(\omega_{2}-\omega_{r}\right)^{3}\right] P\left(J_{r}, J_{2}\right) \\
& +\frac{2 e^{2}}{3 c^{3}} \epsilon^{2} a^{2} \frac{\hbar}{2}\left\{G^{2}\left(\omega_{2}+\omega_{r}\right)^{3}+H^{2}\left(\omega_{2}-\omega_{r}\right)^{3}\right\} \frac{d P\left(J_{r}, J_{2}\right)}{d J_{r}} \\
& +\frac{2 e^{2}}{3 c^{3}} \epsilon^{2} a^{2} \frac{\hbar}{2}\left\{G^{2}\left(\omega_{2}+\omega_{r}\right)^{3}+H^{2}\left(\omega_{2}-\omega_{r}\right)^{3}\right\} \frac{d P\left(J_{r}, J_{2}\right)}{d J_{2}}
\end{aligned}
$$


and the second, labeled by the index 2 , requires

$$
\begin{aligned}
0 & =-A_{2} P\left(J_{r}, J_{2}\right)+B_{2 r} \frac{d P\left(J_{r}, J_{2}\right)}{d J_{r}}+B_{22} \frac{d P\left(J_{r}, J_{2}\right)}{d J_{2}} \\
& =\frac{2 e^{2}}{3 c^{3}}\left\{a^{2} \omega_{2}^{3}+\epsilon^{2} a^{2}\left[G^{2}\left(\omega_{2}+\omega_{r}\right)^{3}+H^{2}\left(\omega_{2}-\omega_{r}\right)^{3}\right]\right\} P\left(J_{r}, J_{2}\right) \\
& +\frac{2 e^{2}}{3 c^{3}} \epsilon^{2} a^{2} \frac{\hbar}{2}\left\{G^{2}\left(\omega_{2}+\omega_{r}\right)^{3}+H^{2}\left(\omega_{2}-\omega_{r}\right)^{3}\right\} \frac{d P\left(J_{r}, J_{2}\right)}{d J_{r}} \\
& +\frac{2 e^{2}}{3 c^{3}} \frac{\hbar}{2}\left\{a^{2} \omega_{2}^{3}+\epsilon^{2} a^{2}\left[G^{2}\left(\omega_{2}+\omega_{r}\right)^{3}+H^{2}\left(\omega_{2}-\omega_{r}\right)^{3}\right]\right\} \frac{d P\left(J_{r}, J_{2}\right)}{d J_{2}} .
\end{aligned}
$$

However, by subtracting one equation from the other and cancelling common factors, the two equations can be reduced to the two differential equations

$$
0=P\left(J_{r}, J_{2}\right)+\frac{\hbar}{2} \frac{d P\left(J_{r}, J_{2}\right)}{d J_{2}}
$$

and

$$
0=\frac{\hbar}{2} \frac{d P\left(J_{r}, J_{2}\right)}{d J_{r}}
$$

The last equation (71) means that (through lowest nonvanishing order in $\epsilon$ ) the probability $P\left(J_{r}, J_{2}\right)$ does not depend upon $J_{r}$, while the solution of (170) is the same as in Eq. (67). This phase space solution (67) indeed works as a solution for both equations (68) and (69). Therefore radiation balance indeed holds through lowest nonvanishing order in $\epsilon$. In this order in $\epsilon$, the lowest order distribution $P\left(J_{2}\right)$ which gives radiation balance at $\omega_{2}$ will also produce radiation balance at $\omega_{2}+\omega_{r}$ and $\omega_{2}-\omega_{r}$.

\section{Marshall and Claverie's Analysis Through First Order in Eccentricity}

In the analysis here, we have used the generalized Fokker-Planck equation of Marshall and Claverie while taking time Fourier expansion coefficients corresponding to the relativistic orbital motion with frequencies $\omega_{2}, \omega_{2}+\omega_{r}$, and $\omega_{2}-\omega_{r}$. However, through first order in the eccentricity $\epsilon$, the relativistic time Fourier coefficients go over to the nonrelativistic coefficients given by Landau and Lifshitz in our Eqs. (31) and (32). Thus we can take the nonrelativistic limits given in Eqs. (46) and (47), and substitute these when calculating the drift and diffusion coefficients in Eqs. (55) through (64). This nonrelativistic limit involves the fundamental frequency $\omega_{2} \rightarrow \omega_{N R}$ of the nonrelativistic orbital motion and its first overtone $\omega_{2}+\omega_{r} \rightarrow 2 \omega_{N R}$. The calculations leading to the resulting phase space 
in Eq. (67) remain valid and radiation balance still holds. Thus Marshall and Claverie's analysis gives radiation balance, involving the particle velocity coefficients through lowest nonvanishing order in the eccentricity $\epsilon$. The failure of radiation balance claimed by Marshall and Claverie involves higher powers in the eccentricity $\epsilon$ where the time Fourier components of the nonrelativistic orbit are not a valid approximation to relativistic electrodynamics.

\section{CONCLUDING REMARKS}

\section{A. Unique Aspects of the Coulomb Potential and Zero-Point Radiation}

The Coulomb potential is part of relativistic electromagnetic theory as is Lorentzinvariant zero-point radiation. The Coulomb potential $V(r)=-e^{2} / r$ is very special in its scaling [28] which gives hydrogen its dependence upon the one parameter of mass $m_{0}$, and leads to the velocity in a circular orbit given by $v=e^{2} / J_{2}$ with no other parameters involved. Zero-point radiation is scale invariant,[28] and combines with the Coulomb potential to give the phase space distribution for circular orbits in Eq. (67) which makes no reference to the frequency of the circular orbital motion. It is this independence from the frequency which allows the radiation balance which we have noted through the lowest nonvanishing order in the eccentricity $\epsilon$.

Any isotropic potential (such as $V(r)=-\alpha / r^{n}$ ) will have circular orbits which have dipole moments connecting the circular orbit of radius $r$ to a single frequency of radiation $\omega(r)$. Thus we should always be able to find an equilibrium phase space associated with circular orbits and some isotropic spectrum $U_{\text {rad }}(\omega)$ of random radiation. However, unlike Eq. (67), these phase space distributions for a general potential and spectrum will depend upon the orbital frequency, and so will not give radiation balance for any distortion of the circular orbital motion.

In addition to the situation of the Coulomb potential and zero-point radiation treated above, there is one other special case where radiation balance holds. Using the MarshallClaverie analysis in the dipole approximation (sketched above), it is easy to show that a multiply periodic mechanical system (29) in the Rayleigh-Jeans spectrum $U_{\text {rad }}(\omega)=k_{B} T$ leads to the Boltzmann distribution $P\left(J_{1}, J_{2}, J_{3}\right)=$ const $\times \exp \left[-U\left(J_{1}, J_{2}, J_{3}\right) /\left(k_{B} T\right)\right]$ for the equilibrium phase space with radiation balance. This calculation was first carried out 
and published in part by van Vleck in 1924. However, this analysis makes no sense for the Coulomb potential where the Boltzmann probability density cannot be normalized. The Coulomb potential of electrodynamics is a very special potential connected to relativity.

\section{B. Symmetries and Radiation Balance}

Our analysis has been very limited in scope. However, there are general principles which encourage the belief that the physical ideas hold more generally. The Coulomb potential is part of Lorentz-invariant classical electrodynamics while classical electromagnetic zero-point radiation has a Lorentz invariant spectrum. How can a relativistic scatterer, such as the relativistic classical hydrogen atom, push the zero-point spectrum toward a spectrum which is not Lorentz invariant? It seems natural to suggest that the Lorentz invariance of the zero-point spectrum must be preserved by relativistic Coulomb scattering, as demonstrated here through lowest nonvanishing order in the orbital eccentricity.

\section{Speculation}

The existence of radiation balance in classical zero-point radiation will hide the electron's radiation emission and also its energy absorption in the classical hydrogen atom. Thus in equilibrium, an observer would not detect net radiation emission or absorption in any frequency range if radiation balance holds. One may speculate that the excited states of atoms correspond to additional configurations at energies above the ground state where radiation balance again exists at least approximately, and that the observed radiation spectra arise from radiation emission by a classical charged particle (electron) when moving from an exited configuration to a lower energy configuration. What is involved is the interaction with random radiation in a non-Markov process where relativity is crucial. If we use nonrela-

tivistic calculations, these calculations must involve valid nonrelativistic approximations to the true relativistic situation in nature. 


\section{Conclusion}

There are four significant messages from the present analysis of the classical hydrogen atom in classical zero-point radiation. 1) In electromagnetism, aspects of relativity may be important even for charged particle motions which have low velocities. 2) Zero-point radiation gives equilibrium for relativistic or nonrelativistic circular Coulomb orbits with a

phase space distribution $P\left(J_{2}\right)=$ const $\times \exp \left[-J_{2} /(\hbar / 2)\right]$ which is independent of the orbital frequency. 3) Through lowest nonvanishing order in the orbital eccentricity $\epsilon$, radiation balance holds for a charged particle in a Coulomb potential in classical zero-point radiation, for both relativistic and nonrelativistic particle motions. 4) In order to avoid self-ionization in elliptical orbits of high eccentricity, relativity may be important for numerical simulations of the classical hydrogen atom in classical zero-point radiation. The message here is very different from the definitive statements regarding the failure of classical electrodynamics which appear in the current modern physics textbooks.

\section{REFERENCES}

[1] See for example, R. Eisberg and R. Resnick, Quantum Physics of Atoms, Molecules, Solids, Nuclei, and Particles 2nd ed. (Wiley, New York 1985); K. S. Krane, Modern Physics, 2nd edn (Wiley, New York 1996); R. Taylor, C. D. Zafiratos, and M. A. Dubson, Modern Physics for Scientists and Engineers, 2nd edn (Pearson, New York, 2003); S. T. Thornton and A. Rex, Modern Physics for Scientists and Engineers, 4th edn (Brooks/Cole, Cengage Learning, Boston, MA, 2013).

[2] T. H. Boyer, "Blackbody radiation in classical physics: A historical perspective," Am. J. Phys. 86, 495-509 (2018).

[3] T. W. Marshall, "Random electrodynamics," Proc. R. Soc. A276, 475-491 (1963).

[4] T. H. Boyer, "Specific heat of a classical, plane, rigid, dipole rotator in electromagnetic zeropoint radiation," Phys. Rev. D 1, 2257-2268 (1970).

[5] T. H. Boyer, "Random electrodynamics: The theory of classical electrodynamics with classical electromagnetic zero-point radiation," Phys. Rev. D 11, 790-808 (1975). 
[6] A review of the work on classical electromagnetic zero-point radiation up to 1996 is provided by L. de la Pena and A. M. Cetto, The Quantum Dice - An Introduction to Stochastic Electrodynamics (Kluwer Academic, Dordrecht 1996). A more recent brief review is given by T. H. Boyer, "Stochastic Electrodynamics: The Closest Classical Approximation to Quantum Theory," Atoms 7(1), 29-39 (2019).

[7] T. H. Boyer, "Equilibrium of random classical electromagnetic radiation in the presence of a nonrelativistic nonlinear electric dipole oscillator," Phys. Rev. D 13, 2832-2845 (1976).

[8] T. H. Boyer, "Statistical equilibrium of nonrelativistic multiply periodic classical systems and random classical electromagnetic radiation," Phys. Rev. A 18, 1228-1237 (1978); “A Connection Between the Adiabatic Hypothesis of Old Quantum Theory and Classical Electrodynamics with Classical Electromagnetic Zero-Point Radiation," Phys. Rev. A 18, 1238-1245 (1978).

[9] T. W. Marshall and P. Claverie, "Stochastic electrodynamics of nonlinear systems. I: Particle in a central field of force," J. Math. Phys. 21, 1819-1925 (1980).

[10] P. Claverie and F. Soto, "Nonrecurrence of the stochastic process for the hydrogen atom problem in stochastic electrodynamics," J. Math. Phys. 23 753-759 (1982).

[11] D. C. Cole and Y. Zou, "Quantum Mechanical Ground State of Hydrogen Obtained from Classical Electrodynamics," Phys. Lett. A 317, 14-20 (2003).

[12] T. M. Nieuwenhuizen and M. T. P. Liska, "Simulation of the hydrogen ground state in stochastic electrodynamics," Phys. Scr. T 165, 014006 (2015).

[13] T. H. Boyer, "Classical Zero-Point Radiation and Relativity; the Problem of Atomic Collapse Revisited," Found. Phys. 46, 880-890 (2016).

[14] T. M. Nieuwenhuizen and M. T. P. Liska, "Simulation of the hydrogen ground state in stochastic electrodynamics-2," Found. Phys. T 45, 1190-1202 (2015).

[15] T. H. Boyer, "Equilibrium for classical zero-point radiation: detailed balance under scattering by a classical charged harmonic oscillator," J. Phys. Commun. 2, 105014(17) (2018).

[16] W. C-W. Huang and H. Batelaan, "Discrete Excitation Spectrum of a Classical Harmonic Oscillator in Zero-Point Radiation," Found. Phys. 45, 333-353 (2015).

[17] M. Born, The Mechanics of the Atom (Ungar, New York 1970), p. 52-53.

[18] See the appendix of the review in ref. 3.

[19] J. D. Jackson, Classical Electrodynamics 3rd ed (Wiley, New York 1999), p.704.

[20] J. H. van Vleck, "The absorption of radiation by multiply periodic orbits, and its relation to 
the correspondence principle and the Rayleigh-Jeans law: Part II. Calculation of absorption by multiply periodic orbits," Phys. Rev. 24, 347-365 (1924); "A correspondence principle for absorption," Jour. Opt. Soc. Amer. 9, 27-30 (1924). See also the much later work by J. H. van Vleck and D. L. Huber, "Absorption, emission, and linewidths: A semihistorical review," Rev. Mod. Phys. 49, 939-959 (1977)..

[21] T. W. Marshall, "Brownian motion and quasi-Markov Processes," Physica 103A, 172-182 (1980).

[22] T. W. Marshall and P. Claverie, "Brownian motion and quasi-Markov Processes II," Physica 104A, 223-232 (1980).

[23] T. W. Marshall, "Statistical Electrodynamics," Proc. Camb. Phil. Soc. 61, 537-546 (1965).

[24] L. D. Landau and E. M. Lifshitz, the Classical Theory of Fields 4th ed(Pergamon, New York, 1985), pp. 181-182. The situation is repeated as homework problem 14.22

in J. D. Jackson's text in ref. 17. Contrary to the suggestions of the texts, the calculation for the radiated power corresponds to electrodynamics only for the fundamental and its first overtone, and only through order $\epsilon^{2}$, and not for the higher powers of $\epsilon$ which are presented.

[25] See ref. 3, p. 800, Eqs. (43) through (47).

[26] H. Goldstein, Classical Mechanics 2nd ed, (Addison-Wesley, Reading, MA 1981), pp.472483. Note that there is a shift in factors of $2 \pi$ between the action-angle variables of the mechanics texts and those used here. Although the designations $J_{r}, J_{\theta}, J_{\phi}$ are common to all the treatments, the designations of $J_{1}$ and $J_{3}$ are interchanged between the treatments in Goldstein and in Born. In the notation of Marshall and Claverie in ref. $7, \xi_{1}=J_{r}$ and $\xi_{2}=J_{2}=J_{\theta}+J_{\phi}$. When the orbit is chosen in the $x y$-plane, then $J_{\theta}=0$ and $J_{2}=J_{\phi}$.

[27] T. H. Boyer, "Unfamiliar trajectories for a relativistic particle in a Kepler or Coulomb potential," Am. J. Phys. 75, 992-997 (2004).

[28] T. H. Boyer, "Scaling symmetries of scatterers of classical zero-point radiation," J. Phys. A: Math. Theor. 40, 9635-9642 (2007). 